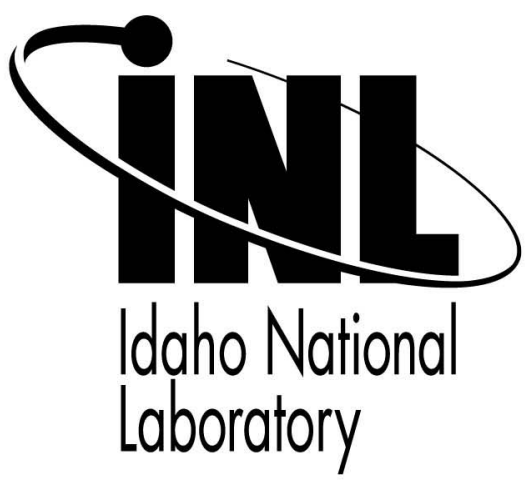

\title{
Radiation Fields in the Vicinity of Compact Accelerator Neutron Generators
}

\author{
NSS-MIC06
}

David L. Chichester

Brandon W. Blackbrun

Augustine J. Caffrey

\section{October 2006}

This is a preprint of a paper intended for publication in a journal or proceedings. Since changes may be made before publication, this preprint should not be cited or reproduced without permission of the author. This document was prepared as an account of work sponsored by an agency of the United States Government. Neither the United States Government nor any agency thereof, or any of their employees, makes any warranty, expressed or implied, or assumes any legal liability or responsibility for any third party's use, or the results of such use, of any information, apparatus, product or process disclosed in this report, or represents that its use by such third party would not infringe privately owned rights. The views expressed in this paper are not necessarily those of the United States Government or the sponsoring agency. 


\title{
Radiation Fields in the Vicinity of Compact Accelerator Neutron Generators
}

\author{
David L. Chichester, Member, IEEE, Brandon W. Blackburn, Member, IEEE and Augustine J. Caffrey, Member, IEEE
}

\begin{abstract}
Intense pulsed radiation fields emitted from sealed tube neutron generators provide a challenge for modern health physics survey instrumentation. The spectral sensitivity of these survey instruments requires calibration under realistic field conditions while the pulsed emission characteristics of neutron generators can vary from conditions of steady-state operation. As a general guide for assessing radiological conditions around neutron generators, experiments and modeling simulations have been performed to assess radiation fields near DD and DT neutron generators. The presence of other materials and material configurations can also have important effects on the radiation dose fields around compact accelerator neutron generators.
\end{abstract}

\section{INTRODUCTION}

$\mathrm{N}$ eutron generators are finding increasing application in active interrogation systems for homeland security screening [1] - [5]. These devices provide a convenient source of fast neutrons which, because they can be turned off, offer an inherent level of safety beyond that present when radioisotopic neutron sources are used. In addition, these devices are generally capable of generating more intense, higher energy neutron fluxes than encountered with typical radiochemical sources. Two practical consequences of these differences from radiochemical sources are that the relative location of a radiological 'safe' perimeter can be significantly further away and the composition and energy of neutron and photon radiation fields can be different.

Unfortunately, modern health physics instrumentation is not necessarily well suited for conducting site surveys in the vicinity of a neutron generator. Most neutron measuring health physics instrumentation is designed and calibrated for measuring either thermal neutron radiation or fission spectrum neutron fields (such as from the spontaneous fission of ${ }^{252} \mathrm{Cf}$ or from the complex neutron emission spectrum of an ${ }^{241} \mathrm{Am}-$ ${ }^{9} \mathrm{Be}(\alpha, \mathrm{n})$ neutron source). In contrast, sealed tube neutron generators produce nearly monoenergetic neutrons at either $2.5 \mathrm{MeV}$ (using the deuterium + deuterium (DD) fusion reaction) or $14.1 \mathrm{MeV}$ (using the deuterium + tritium (DT) fusion reaction) by accelerating ions of deuterium and/or tritium into metal hydride targets which also contain deuterium and/or tritium. Similarly, most photon measuring survey instruments are designed and calibrated for

Manuscript received November 17, $2006 . \quad$ Idaho National Laboratory is operated for the U.S. Department of Energy by Battelle Energy Alliance under DOE contract DE-AC07-05ID14517.

D. L. Chichester, B. W. Blackburn, and A. J. Caffrey are with Idaho National Laboratory, Idaho Falls, ID 83415-3840 USA (telephone: 208-5268920; e-mail: David.Chichester@INL.gov; Brandon.Blackburn@INL.gov; Gus.Caffrey@INL.gov). measurements at photon energies less then about $2 \mathrm{MeV}$ while photon fields resulting from fast neutron inelastic scattering and prompt nuclear decay often contain photons $>2 \mathrm{MeV}$. Another important complication when measuring both neutron and photon fields from neutron generators arises from the fact that many applications require pulsed mode operation. Depending upon the neutron emission rate of the neutron generator and the location where the measurements are taken it is possible for these devices to suffer dead-time loses when short duration (less then $\sim 10 \mu \mathrm{s}$ ) pulses are generated by the device.

Several papers have been written which benchmark the performance of modern commercial neutron health physics instruments [6] - [10]. Usually they have response profiles (response per ambient dose equivalent) which differ only 5$10 \%$ in the energy range of $1-10 \mathrm{MeV}$. At energies less then 1 $\mathrm{MeV}$ variations in sensitivity among different commercial instruments vary by a factor of 2-3. At energies greater then $10 \mathrm{MeV}$ the response of older generation instruments quickly falls off while more modern instruments, specifically designed for use at higher energies, continue to demonstrate comparable responses for neutron energies up to several hundred $\mathrm{MeV}$. Since these devices are rarely calibrated in monoenergetic neutron fields it is important to understand the radiation fields which might be expected near these devices. For example, the SWENDI-II neutron rem meter, when calibrated in a ${ }^{252} \mathrm{Cf}$ radiation field, has a side-on measured sensitivity of $45.7 \mathrm{cpm} /(\mu \mathrm{Sv} / \mathrm{h})(457 \mathrm{cpm} /(\mathrm{mrem} / \mathrm{h}))$ (with dose measured in $\mathrm{H}^{*}(10)$ dose equivalent) [6]. However, the measured sensitivity of the same instrument for $2.5 \mathrm{MeV}$ neutrons is $50.5 \mathrm{cpm} /(\mu \mathrm{Sv} / \mathrm{h})$ while the sensitivity for 14.8 $\mathrm{MeV}$ neutrons has been measured to be $28.8 \mathrm{cpm} /(\mu \mathrm{Sv} / \mathrm{h})$ [6]. Therefore, in a facility using a SWENDI-II calibrated for ${ }^{252} \mathrm{Cf}$, for example, unshielded neutron dose rates near a DD neutron generator would be over reported by an additional $11 \%$ while unshielded neutron dose rates near a DT generator would be underreported at $63 \%$ of the true value.

This paper presents data from modeling and experiments focused towards understanding radiation fields near compact accelerator neutron generators, evaluating the impact of fluxto-dose conversion coefficients, neutron scattering off of concrete floors, walls and ceilings, and estimating the effects small amounts of shields can have on DD and DT neutron spectra. Concerning neutron rem meter instrument response, experimental results are offered illustrating the differences between calculated and measured neutron dose rates near a DD neutron generator. This information is intended to provide a reference for understanding and estimating potential radiation fields around neutron generators for facility 
operations planning and when direct measurements can not be taken.

\section{MODELING AND EXPERIMENTS}

Often new users of neutron generators face logistical hurdles with radiological safety staff at their institutions as they seek permission to operate these devices. In most cases health physics staff are not familiar with these instruments, their background experience related to neutron shielding is focused towards fission neutrons and thermal neutrons, and previous experiences with accelerators (if applicable) if in the realm of very large, high radiation dose rate machines such as high energy physics accelerators or medical cyclotrons. Because of this it is not uncommon for users to spend several months working with their radiation safety coordinators to develop safe operating procedures which are applicable for use with neutron generators (recognizing that they can be "turned-off", left unattended while operating if in a secure location or when in the off state, and noting that their radiation output is significantly different from reactors or radioisotope sources such as Cf-252).

To provide background information for assistance in understanding the radiation fields from neutron generators calculations and numerical modeling have been performed to assess a variety of conditions. In section A neutron dose rate versus distance from a DT point source is assessed based upon pure $1 / \mathrm{r}^{2}$ relationships (no scattering) and for a source located $1 \mathrm{~m}$ above a concrete pad (including scattering), comparing the use of NCRP and ICRP recommended flux-to-dose conversion guidelines. In section $\mathrm{B}$ results are presented for simulations evaluating dose rate versus distance from a DT neutron source, located $1 \mathrm{~m}$ above a concrete pad (including scattering), for different arrangements of nearby scattering walls and ceilings. Section $\mathrm{C}$ evaluates the spectra of neutrons from DD and DT neutron generators transmitted through $10 \mathrm{~cm}$ shields made of a variety of materials found in nuclear physics laboratories. To address the important issue of health physics instrument response to monoenergetic neutrons section D presents comparisons of calculated and measured neutron dose rates near a DD neutron generator.

Nuclear simulations presented in this paper were performed using the Monte Carlo code package MCNP 5 [11]. Neutron flux values were tallied in $0.5 \mathrm{MeV}$ bins from 0 to $16 \mathrm{MeV}$ at a distance of $100 \mathrm{~cm}$ from the point source. Process times were chosen to be long enough to ensure that statistical uncertainties from the Monte Carlo process were less then $1 \%$ in all cases.

\section{A. Neutron Dose Rate and Flux-to-Dose Conversions}

One method used to evaluate dose rates near neutron generators is to calculate the neutron flux and spectrum at the position(s) of interest and then to use published flux-to-dose conversion factors to translate this information to dose rates. This technique if generally very useful for the evaluation of radiation fields for health physics and safety analysis, although more precise techniques are also available which are used in the medical physics community. A set of neutron flux-to-dose conversion rates commonly accepted for use for this purpose is the NCRP-38 -- ANSI/ANS-6.1.1-1977 conversion set, another is the other is the ICRP-21 set [12]. To compare these conversion sets calculations were performed to evaluate neutron dose rate versus distance relationships using both the NCRP-38 and ICRP-21 without neutron scattering (simply expanding the DT neutron flux versus $1 / \mathrm{r}^{2}$ scaling) and with neutron scattering (using MCNP 5). For these calculations a monoenergetic isotropic DT point source of neutrons, located 1 meter above a concrete pad, was used as the source term; neutron flux was computed using MCNP 5 , at varying distances from this source at a height of 1 meter from the floor, and then converted to dose using the above mentioned conversion factors.

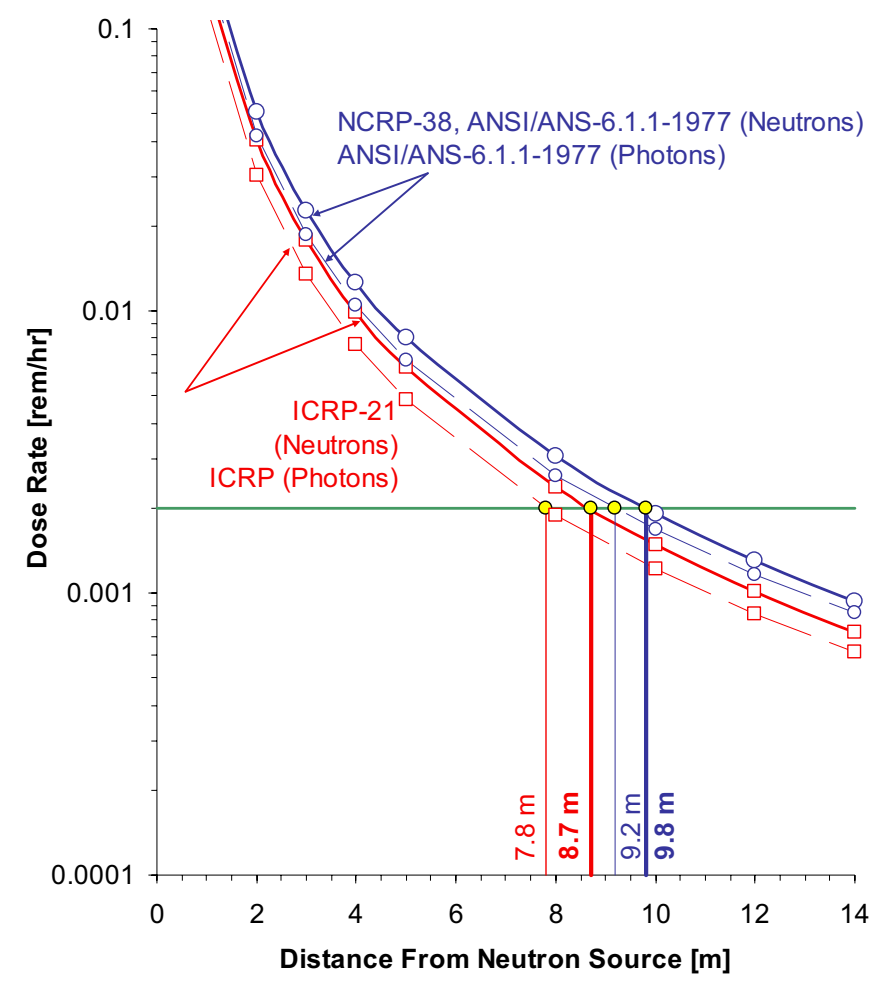

Fig. 1 Comparison of dose rate versus distance estimated using the NCRP (circles) and ICRP (squares) conversion factors for a $1 \times 10^{8} \mathrm{n} / \mathrm{s}$ DT neutron source 1 meter above a concrete pad without scattering (dashed lines, calculated using $1 / \mathrm{r}^{2}$ scaling) and with scattering (solid lines, calculated using MCNP 5).

As shown in Fig. 1 localized neutron scattering of DT neutrons (comparing solid and dashed lines) from a concrete pad plays a small but measurable role in boosting the radiation field around the DT neutron source. Similarly, the choice of using one or the other of the two conversion sets can also lead to a different interpretation of the radiation fields around the source. Combing these factors a relative uncertainty in the evaluation of the $2 \mathrm{mrem} / \mathrm{hr}$ dose boundary can be up to $2 \mathrm{~m}$, or $25 \%$ in the extreme when the ICRP values are used with $1 / \mathrm{r}^{2}$ scaling in comparison with the neutron scattered field analyzed using the NCRP set (which leads to $\sim 3.5 \mathrm{mrem} / \mathrm{hr}$ at $7.8 \mathrm{~m}$ ). This analysis may have important implications with regard to the establishment of radiation safety boundaries for personnel working near neutron generators. Although the 
actual impact of the discrepancies shown here is small, $\sim 1.5$ $\mathrm{mrem} / \mathrm{hr}$, in situations where regulatory compliance is being ascertained, or in situations where personnel are working closer to the neutron source or are working with higher intensity sources, these details might be significant.

\section{B. Neutron Dose Rates Near Concrete Walls \& Floors}

Having examined the important impact flux-to-dose conversion factor selection can have when determining radiation fields near neutron generators, and the effect neutron scattering from a floor can have to increase these fields above what $1 / r^{2}$ scaling would otherwise yield, further simulations were performed to examine the effects of other scattering surfaces. Specifically, cases were examined i) where a $2 \mathrm{~m}$ tall, $2 \mathrm{~m}$ thick, infinitely long concrete wall was located $1 \mathrm{~m}$ behind the source (relative to the direction in which dose is calculated) ("Rear Reflecting Wall"), ii) where the same wall was located $1 \mathrm{~m}$ to the side of the source ("Side Reflecting Wall"), iii) where an infinite concrete ceiling $2 \mathrm{~m}$ thick was located $2 \mathrm{~m}$ above the floor ("Ceiling"), and iv) where the source was located in a $2 \mathrm{~m} \times 2 \mathrm{~m}$ concrete corridor and neutron dose rates were evaluated along the length of the corridor from the source ("Corridor"). Plots of dose rate versus position for these four cases, and the base line case of a simple concrete pad, are presented in Fig. 2 where a $1 \times 10^{8}$ $\mathrm{n} / \mathrm{s}$ monoenergetic isotopic DT neutron source was used together with the NCRP-38 flux-to-dose conversion values.

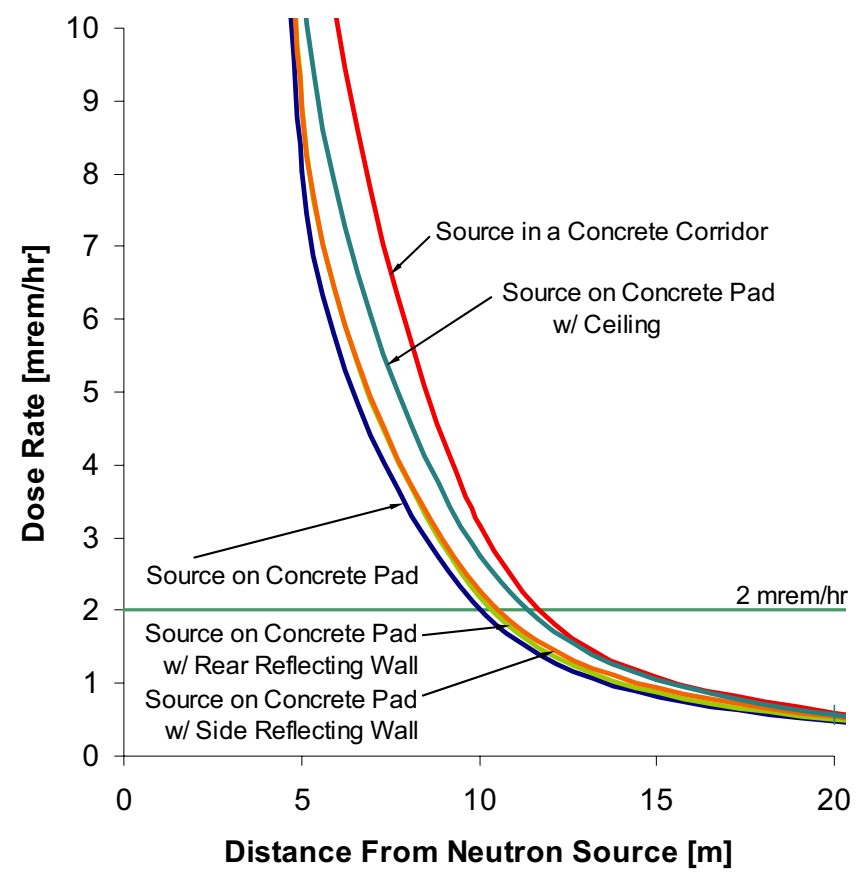

Fig. 2 Comparison of dose rate versus distance estimated using the NCRP conversion factors for a $1 \times 10^{8} \mathrm{n} / \mathrm{s}$ DT neutron source 1 meter above a concrete pad with options including a rear concrete wall, a side concrete wall, a concrete ceiling and for the case when the source is located in the center of a concrete corridor (calculated using MCNP 5).
For these different scenarios it is again evident that neutron scattering can have an important effect on the magnitude of the external radiation field in the vicinity of monoenergetic fusion neutron sources. These situations are important because of the increasing application of compact accelerator neutron generators in field work where operations may take place without the ability to formally map dosimetry radiation contour fields are when operators are not equipped to perform these tasks. When operating in a parking garage, for example, the standard $2 \mathrm{mrem} / \mathrm{hr}$ boundary line might be as far as 11.5 $\mathrm{m}$ from the source. If in this situation simple $1 / \mathrm{r}^{2}$ scaling was used to determine the $2 \mathrm{mrem} / \mathrm{hr}$ location the boundary might be marked as close as $7.8 \mathrm{~m}$ (if the ICRP conversion set is used); in this instance the actual dose rate at the marked boundary might be as high as $6 \mathrm{mrem} / \mathrm{hr}$. Although this is still a relatively low exposure rate it is 3 times higher then the reference $2 \mathrm{mrem} / \mathrm{hr}$ rate. In regulatory situations or when operating at closer to the generator or at higher yields this could become an important ALARA consideration.

\section{Neutron Spectra Through $10 \mathrm{~cm}$ Shields}

Often times application developers will surround neutron generators with small amounts of materials in an effort custom tailor the flux from the devices for their specific project. Different materials can have noticeable changes on the neutron spectrum from the generators in these cases. To examine this numerical modeling has been performed using MCNP 5 to evaluate the spectra of DD and DT neutrons transmitted through shields of different materials. The materials evaluated in this work included cement, water, polyethylene, beryllium, a composite mixture of polyethylene with bismuth (density $=3 \mathrm{~g} / \mathrm{cm}^{3}, 81 \%$ bismuth by weight), lead, and bismuth. These materials are representative of what is frequently used with compact neutron generators to tailor neutron spectra, attenuate neutrons to shield nearby detectors, and for moderating and reflecting neutrons. The model consisted of an isotropic point source centered within a sphere of the shield material having a radius of $10 \mathrm{~cm}$, spectra were determined at a distance of $1 \mathrm{~m}$ from the source. No nearby neutron scattering was considered in this analysis. Spectra for DD source neutrons are presented in Fig. 3, spectra for DT source neutrons are presented in Fig. 4.

Typical low- $Z$ shield materials including water, polyethylene (poly), and cement have comparable effects on the transmitted neutron energy profile, producing a relatively smooth continuum in the $0-10 \mathrm{MeV}$ range. This can be important in fast neutron inelastic scattering measurements, for example, in order to ensure a uniform neutron distribution for exciting prompt states in materials. In higher $\mathrm{Z}$ materials, and $\mathrm{Be}$, with DT neutrons the $(n, 2 n)$ and $(n, \alpha)$ reaction channels lead to significant neutron multiplication. These materials can be used to increase the lower energy neutron flux near neutron generators while more efficiently removing/depleting the MeV-range neutron intensity. Because of this these materials are particularly well suited for shielding co-located radiation detectors from nearby neutron generators, protecting them from the radiation damage that 
can occur in some detector materials as a result of prolonged exposure to neutron radiation.
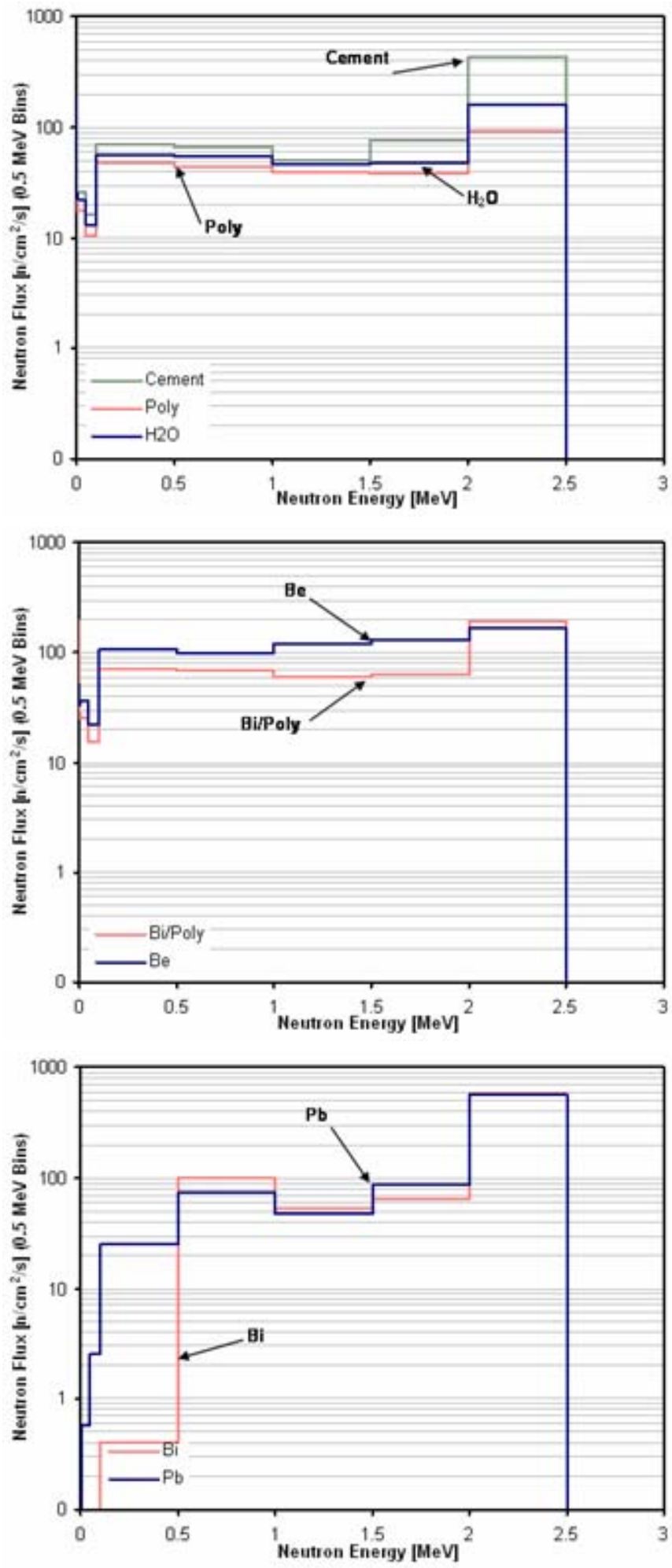

Fig. 3 Spectra resulting from the transmission of DD neutrons through 10 cm thick shields of: cement, water, and polyethylene (top); beryllium and a composite mixture of polyethylene with bismuth (density $=3 \mathrm{~g} / \mathrm{cm}^{3}, 81 \%$ bismuth by weight) (middle), and lead and bismuth (bottom).
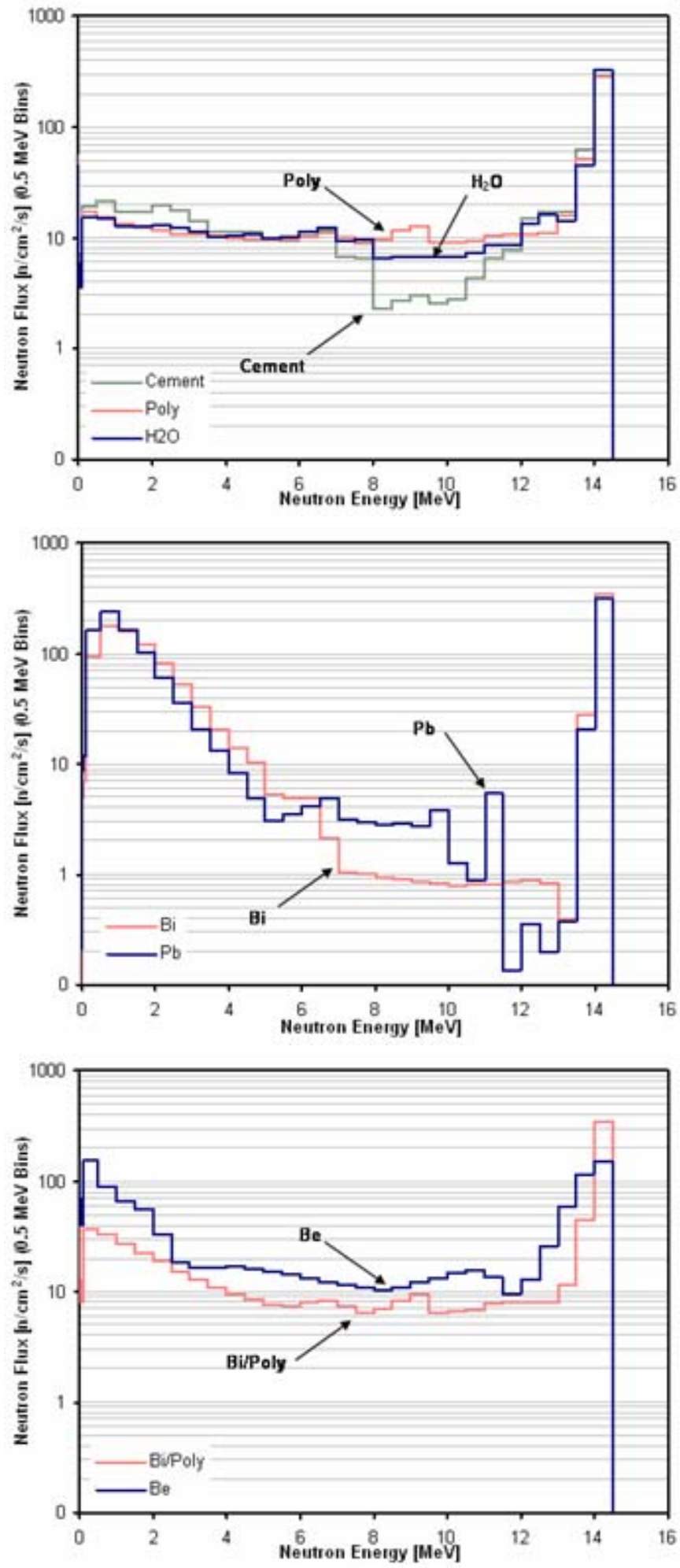

Fig. 4 Spectra resulting from the transmission of DT neutrons through 10 cm thick shields of: cement, water, and polyethylene (top); beryllium and a composite mixture of polyethylene with bismuth (density $=3 \mathrm{~g} / \mathrm{cm}^{3}, 81 \%$ bismuth by weight) (middle), and lead and bismuth (bottom). 


\section{Health Physics Instrument Response to DD and DT Neutrons}

To illustrate the importance of considering instrument calibration, energy sensitivity and local neutron scattering in determining neutron dose rates near compact accelerator neutron generators an experiment was performed to measure the neutron dose rate near a commercial DD neutron generator (Model: MP320, Manufacturer: Thermo Electron Corporation, Colorado Springs, CO, USA). The generator was placed upon a steel table so that the neutron production target was located $1 \mathrm{~m}$ above a concrete floor. Nearby walls were greater then 2 $\mathrm{m}$ from the generator, comprised of one course of hollow cement masonry block. The ceiling was approximately $4 \mathrm{~m}$ from the floor, comprised of an approximately $15 \mathrm{~cm}$ thick concrete pad which served as the floor for the second level of the two-story building. A cylindrical SWENDI-II neutron rem meter (Manufacturer: Thermo Electron Corporation, Santa Fe, NM, USA) was placed on a separate steel table, oriented with its side centered at the height of the neutron production target with a distance of 1 meter from the neutron target to the center of the instrument. On both sides of the SWENDI-II on this table were additional polyethylene moderator neutron rem meters; it is estimated that these nearby reflectors served to enhance the SWENDI-II response by $10 \%$. A general plot of the instruments monoenergetic response profile, as determined by Olsher et al. in [6], is provided in Fig. 5 together with reported response profiles for bare and shielded ${ }^{252} \mathrm{Cf}$ neutrons, ${ }^{241} \mathrm{AmBe}$ neutrons and the vendors nominal in-house calibration response. The instrument used for the experiments described here had been calibrated at the Health Physics Instrument Laboratory (HPIL) at Idaho National Laboratory using unmoderated ${ }^{252} \mathrm{Cf}$ sources within the previous 6 months.

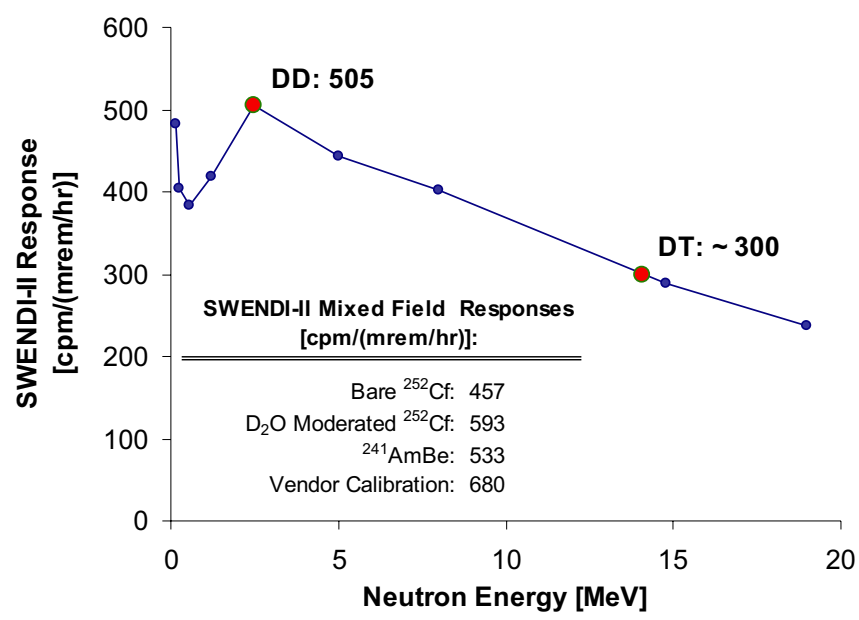

Fig. 5 Response sensitivity of the SWENDI-II neutron rem meter as a function of incident neutron energy; the inset table lists the instruments response sensitivity to bare and moderated ${ }^{252} \mathrm{Cf}$ neutron radiation, an ${ }^{241} \mathrm{AmBe}$ reference field, and the vendors calibration response [6].

The neutron generator was operated in a steady-state output mode without pulsing, the accelerating high voltage level was set at $90 \mathrm{kV}$ and the beam current was set at $60 \mu \mathrm{A}$. At these settings the neutron flux on the outside of the generator housing was measured using indium foil activation. The mass of the $99.99 \%$ pure foil used for this measurement was $5.11 \pm$ $0.05 \mathrm{~g}$, it's thickness was $0.23 \pm 0.01 \mathrm{~cm}$. The foil was placed directly to the side of the generator's neutron target, at $90^{\circ}$ with respect to the ion beam impinging upon the generators internal target. Although neutron dose rate measurement using the SWENDI-II took only a few minutes the activation measurement for the In foil lasted for 60 minutes. After irradiation the foil was quickly moved to a calibrated counting station using an HPGe detector were it was subsequently analyzed for 60 minutes. Indium activity was determined using the $336.24 \mathrm{keV}$ gamma ray from the ${ }^{115} \operatorname{In}\left(\mathrm{n}, \mathrm{n}^{\prime}\right)^{115 \mathrm{~m}} \mathrm{In}$ activation product. Corrections were made for selfattenuation in the sample. The fast neutron flux was determined to be $4388 \pm 705 \mathrm{n} / \mathrm{cm}^{2} / \mathrm{s}$. Accounting for the distance from the foil to the center of the neutron generator the effective neutron generator yield was estimated to be $1.1 \mathrm{x}$ $10^{6} \pm 0.4 \times 10^{6}(2-\sigma) \mathrm{n} / \mathrm{s}$. Extrapolating this yield to a distance of 1 meter to determine neutron flux and then using the NCRP-38 - ANSI/ANS-6.1.1-1977 flux-to-dose conversion for DD neutrons, the neutron dose rate $1 \mathrm{~m}$ from the generator was calculated to be $1.1 \pm 0.4(2-\sigma) \mathrm{mrem} / \mathrm{hr}$. The neutron dose rate measured by the SWENDI-II was $2.7 \pm$ $0.3(2-\sigma) \mathrm{mrem} / \mathrm{hr}$.
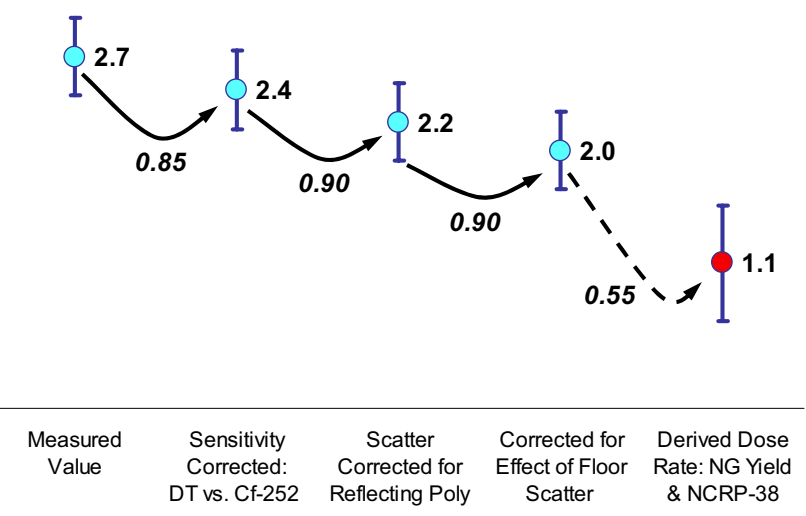

Fig. 6 Bridge chart illustrating corrections to equate the difference between neutron dose rate measured near a DD neutron generator (left, $2.7 \pm 0.3(2-\sigma)[\mathrm{mrem} / \mathrm{hr}])$ and the calculated dose rate (right, $1.1 \pm 0.4(2-$ $\sigma)[\mathrm{mrem} / \mathrm{hr}]$ ) for a DD point source emitting $1.1 \times 10^{6} \mathrm{n} / \mathrm{s} \pm 0.4 \times 10^{6} \mathrm{n} / \mathrm{s}$ (2- $\sigma$ error).

In an attempt to reconcile these values a bridge chart has been developed to illustrate the effect of some known correction factors (Fig. 6). Starting with the instruments reported value on the left one correction can be made for the difference in sensitivity between DD neutrons and a broad ${ }^{252} \mathrm{Cf}$ neutron spectrum by taking the ratio $457 / 505=0.9$. A second correction factor can be taken to account for the presence of the additional reflecting material to the sides of the SWENDI-II, 0.9. A third correction for reconciling the measured and calculated neutron dose rates can be taken to correct for neutron scattering from the floor, from Monte Carlo modeling, by using a factor of 0.9. Taken together the 
final gap between measured value and calculated value remains at $0.9 \mathrm{mrem} / \mathrm{hr}$.

One contributing cause to help explain the remaining discrepancy might be the anisotropic nature of neutron emission from the DD reaction [13]. The neutron yield in these measurements was measured at $90^{\circ}$ to the generator's ion beam; however, for DD neutrons with an energy of roughly $50 \mathrm{keV}$ (appropriate for a $90 \mathrm{keV}$ accelerator with a mostly molecular $\mathrm{D}_{2}$ ion beam) the neutron yield at this angle is roughly $25 \%$ less then the generator's total output. Other correction factors might include drift in the SWENDI-II since its calibration, the not-accounted-for effects of neutron scattering from the ceiling and walls, and errors associated with the efficiency calibration for the HPGe detector used to analyze the indium foil.

\section{SUMMARY}

Many phenomena can act to complicate the assessment of radiation fields in the vicinity of compact accelerator neutron generators. Concerning health physics planning and radiation dose profiling the choice of flux-to-dose conversion factors can lead to different estimates of neutron dose rates, possibly leading to important discrepancies between different radiological assessments when different conversion values are used. When using consistent conversion values, of which the NCRP-38 - ANSI/ANS-6.1.1-1977 appear to be more conservative of the two considered here, differences between ideal non-scattered and scattered radiation profiles must be considered. The importance of neutron scattering off of floors, nearby walls and ceilings can serve to significantly alter actual radiation fields from calculations of fields based upon simple $1 / \mathrm{r}^{2}$ scaling rules from the neutron source strength. Complicating reactions in some materials including the $(n, 2 n)$ and $(n, \alpha)$ reactions can also obscure the understanding of radiation profiles near neutron generators. These effects can be used to a degree to custom tailor DD and DT radiation profiles to suit particular circumstances, such as flattening neutron energy spectra in the $\mathrm{MeV}$ range or optimally shielding sensitive equipment located near fusion neutron sources. Finally, to fully understand measurements of unshielded DD and DT radiation using standard health physics instrumentation, which are commonly calibrated to fission neutron spectra, one must carefully account for the nonlinear energy response most instruments exhibit for fast neutrons and the effects of neutron scattering from nearby objects.

\section{REFERENCES}

[1] Eberhardt, J. E., Rainey, S., Stevens, R. J., Sowerby, B. D., and Tickner, J. R., "Fast neutron radiography scanner for the detection of contraband in air cargo containers," App. Rad. Iso. 63, pp. 179-188, 2005.

[2] Aleksandrov, V. D., et al., "Application of neutron generators for high explosives, toxic agents and fissile material detection," App. Rad. Iso. 63, pp. 537-543, 2005.

[3] Strellis, D. and Gozani, T., "Classifying threats with a $14-\mathrm{MeV}$ neutron interrogation system," App. Rad. Iso. 63, pp. 799-803, 2005.

[4] Buffler, A., "Contraband detection with fast neutrons," Rad. Phys. Chem. 71, pp. 853-861, 2004)
[5] Slaughter, D., et al., "Detection of special nuclear material in cargo containers using neutron interrogation," Report UCRL-ID-155315, Lawrence Livermore National Laboratory, Livermore, CA, USA.

[6] Olsher, R. H., Hsu, H.-H., Beverding, A., and Kleck, J. H., "WENDI: An improved neutron rem meter," Report LA-UR-99-6551, Los Alamos National Laboratory, Los Alamos, NM, USA, 1999.

[7] Olsher, R. H., et al., "PRESCILA: A new, lightweight, neutron rem meter," Report LA-UR-03-2638, Los Alamos National Laboratory, Los Alamos, NM, USA, 2003.

[8] Fehrenbacher, G., Gutermuth, F., and Radon, T., "Active neutron dose meters for the application at high energy particle accelerators," GSI 2003 Annual Scientific Report, pp. 221-222, Gesellschaft für Schwerionenforschung, Garmstadt, Germany, 2003.

[9] Morris, R. L., "Rapid measurement of neutron dose rate for transport index," Report RFP-5272, Rocky Flats Environmental Technology Site , Golden, CO, USA, 2000.

[10] Birattari, C., Ferrari, A., Nuccetelli, C., Pelliccioni, M., and Silari, M., "An extended range neutron rem counter," Nucl. Inst. Meth. Phys. Res. A 297, pp. 250-257, 1990.

[11] X-5 Monte Carlo Team, "MCNP - A General Monte Carlo N-Particle Transport Code, Version 5," Report LA-UR-03-1987, Los Alamos National Laboratory, Los Alamos, NM, USA (2005).

[12] As referenced in [11].

[13] "Handbook of Fast Neutron Generators", Csikai, J., ed., CRC Press, Boca Raton, FL, USA (1987) p. 10. 